\title{
Comparing different software packages for the mapping of strain from scanning precession diffraction data
}

Ian MacLaren ${ }^{1}$, Emma Devine ${ }^{2}$, Hristo Gergov², Gary Paterson ${ }^{3}$, Harikrishnan K. P. ${ }^{4}$, Benjamin Savitzky $^{5}$, Colin Ophus ${ }^{5}$, Renliang Yuan ${ }^{6}$, Jian-Min Zuo ${ }^{7}$, Kirsten Forster ${ }^{2}$, Gaja Kobe ${ }^{2}$, Elizabeth Koppany $^{2}$, Kirsten McClymont ${ }^{2}$, Anjelo Narendran ${ }^{2}$ and David Riley ${ }^{2}$

${ }^{1}$ University of Glasgow, United States, ${ }^{2}$ University of Glasgow, United Kingdom, ${ }^{3}$ University of Glasgow, Glasgow, Scotland, United Kingdom, ${ }^{4}$ School of Applied and Engineering Physics, Cornell University, Ithaca, NY, USA, Ithaca, New York, United States, ${ }^{5}$ Lawrence Berkeley National Laboratory, California, United States, ${ }^{6}$ University of Illinois at Urbana-Champaign, Illinois, United States, ${ }^{7}$ University of Illinois at Urbana-Champaign, Urbana, Illinois, United States

Mapping strain in materials using electron microscopy has been accomplished by a wide range of techniques applicable over a wide range of length-scales from nanometres to microns. These include higher order Laue zone line measurements in convergent beam electron diffraction, measurement of atomic plane separations using atomic resolution imaging, dark field electron holography, and scanning nanobeam electron diffraction (with or without precession of the electron beam).

With the advent of pixelated direct electron detectors which read out at $\mathrm{kHz}$ frequencies, it has recently become possible to scan large areas of samples at high spatial resolution using such techniques and still generate high quality, low noise datasets for analysis. In principle, if the diffraction spots are clearly resolved, then measurement of the spot spacings in a pattern should provide a high precision measurement of any changes in lattice parameter and thus allow strain mapping. There have been a number of publications over the last decade or so on this topic. Additionally, many of the more recent publications on the topic have been associated with Open Source software published in accessible internet sites, making it easy for other groups to use them.

It is, however, the case, that there has been no systematic evaluation or comparison of these codes. This work applies a range of codes to the same two datasets, which show slightly different features. Both were recorded on a JEOL ARM $200 \mathrm{~F}$ at $200 \mathrm{kV}$ using the smallest objective aperture to produce a convergence semiangle of $1.3 \mathrm{mrad}$ giving a probe size of the order of $2 \mathrm{~nm}$ and using a beam current of about $14 \mathrm{pA}$, and then using scanning precession electron diffraction controlled by a TopSpin/Digistar acquisition system (NanoMegas SPRL) coupled to a MerlinEM direct detection system (Quantum Detectors Ltd.) using a precession tilt of $0.5^{\circ}$ and 1 precession per pattern (with $0.01 \mathrm{~s}$ per pixel).

The codes used to evaluate the data are as follows:

1. TopSpin Strain Analysis - commercial software from Nanomegas ${ }^{1,2}$ - using disc cross correlation

2. FPD from the University of Glasgow ${ }^{3}$ - using edge detection and cross correlation

3. py4DSTEM from Lawrence Berkeley National Laboratory ${ }^{4}-$ using cross correlation to a template

4. imToolBox from University of Illinois Urbana-Champaign ${ }^{5}-$ using edge detection and Hough transformation

5. PC-STEM from Cornell University ${ }^{6}$ - using Fourier transformation of the diffraction patterns to real space to determine the periodicity of the whole pattern

The first four codes should in principle be similar in that they detect the disc positions, calculate a centre and then calculate a lattice from all the disc positions and compute strain from that. Any differences will be down to the details of the routines for determining disc positions. All these work well with a reasonable number of diffraction spots present in the Zero order Laue zone, and all well-resolved. The final one is better optimised for shorter camera lengths to give larger spacings in the Fourier transform, and the data 
taken in this study is not optimised for that. A future study will record datasets at different camera lengths from the same area for a fairer comparison.

The first dataset is from a thin film of a perovskite oxide (nominally $\mathrm{La}_{2} \mathrm{MnNiO}_{6}$ ) grown on (001) $\mathrm{SrTiO}_{3}$ where the film should naturally have a smaller lattice parameter than the substrate, so we expect a strain distribution but possibly not an instant switch from one lattice parameter to the other. The raw dataset was of $188 \times 17$ pixels, each of $2 \mathrm{~nm}$ in size although the top two rows pixels have been cropped as this was mostly surface and protective platinum. It is shown in Figure 1 that all tested codes show a similar behaviour in this case, although there are differences in how much noise comes through into the final maps, in the sharpness of the switch at the interface, and in the behaviour around a $\mathrm{NiO}$ inclusion on the right. Sample single diffraction patterns are shown from film (from the black reference pixel) and substrate (orange marker in the top map) to show the raw data used in the calculations - these were processed by applying a power of 0.25 to the pixel values to enhance weak diffraction spots.

The second dataset is from a quantum well structure with a periodicity less than $10 \mathrm{~nm}$ in $\mathrm{Ge}_{-} \mathrm{SiGe}^{7}$ where strain should vary rapidly on the nanoscale and is of $155 \times 16$ pixels of $1 \mathrm{~nm}$ in size. In this case Figure 2 shows that there are more marked differences between three of codes although the same rapid variation is seen in all cases. Specifically, the average strain value is not computed by all codes away from the single composition buffer (right) into the quantum well structure (left).

Work is underway to understand the reasons for these discrepancies, but it is clear that the different approaches to quantifying the diffraction pattern periodicities make a significant and important difference to the results, and the presentation will present advances in understanding this and the implications for accurate strain measurement in nanobeam electron diffraction.

Acknowledgements

The authors are grateful to Dr WW Li and Prof. Judith L. Driscoll at the University of Cambridge for the provision of the perovskite thin film sample. The sample used for the Quantum well measurements was previously described in Bashir et al. ${ }^{7}$, which gives more information on the physical and chemical structure of this heterostructure. We are indebted to all the help received from Nanomegas (and especially Dr Stavros Nicolopoulos) in the installation and optimisation of the prototype Scanning Precession Electron Diffraction System integrating both the MerlinEM direct detection system, as well as discussions on the TopSpin strain analysis package (especially with Amith Darbal and Jing Lu). 


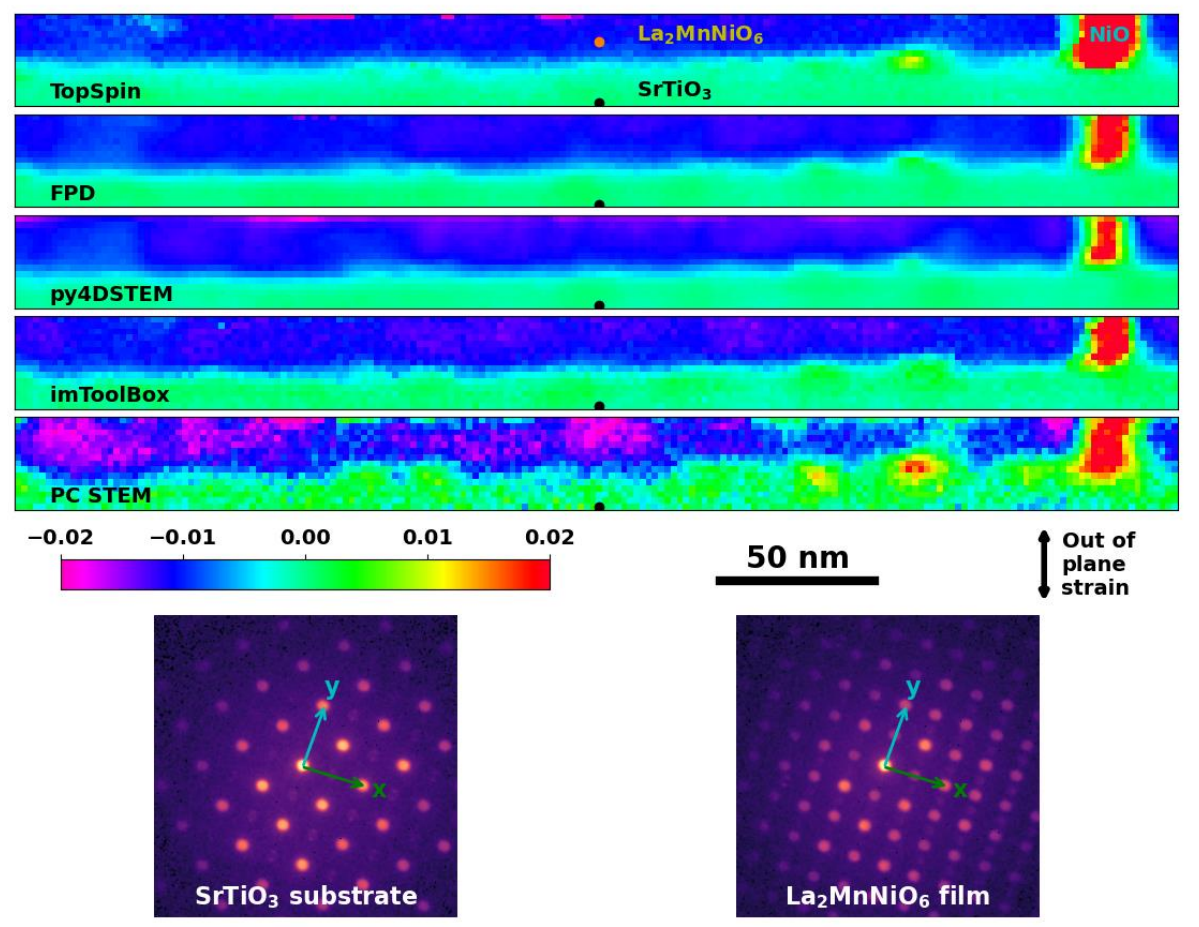

Figure 1. Out-of-plane strain maps calculated from the same dataset using five different software codes from an area of $188 \times 15$ pixels of $2 \mathrm{~nm}$ in side across a thin film of $\mathrm{La}_{2} \mathrm{MnNiO}_{6}$ grown on (001) $\mathrm{SrTiO}_{3}$ by pulsed laser deposition. A NiO inclusion is seen on the far right and the large lattice parameter results in saturation of the strain measurements in this region (on the colour scale used). The black dot on each map shows the pixel used as the zero strain reference. 


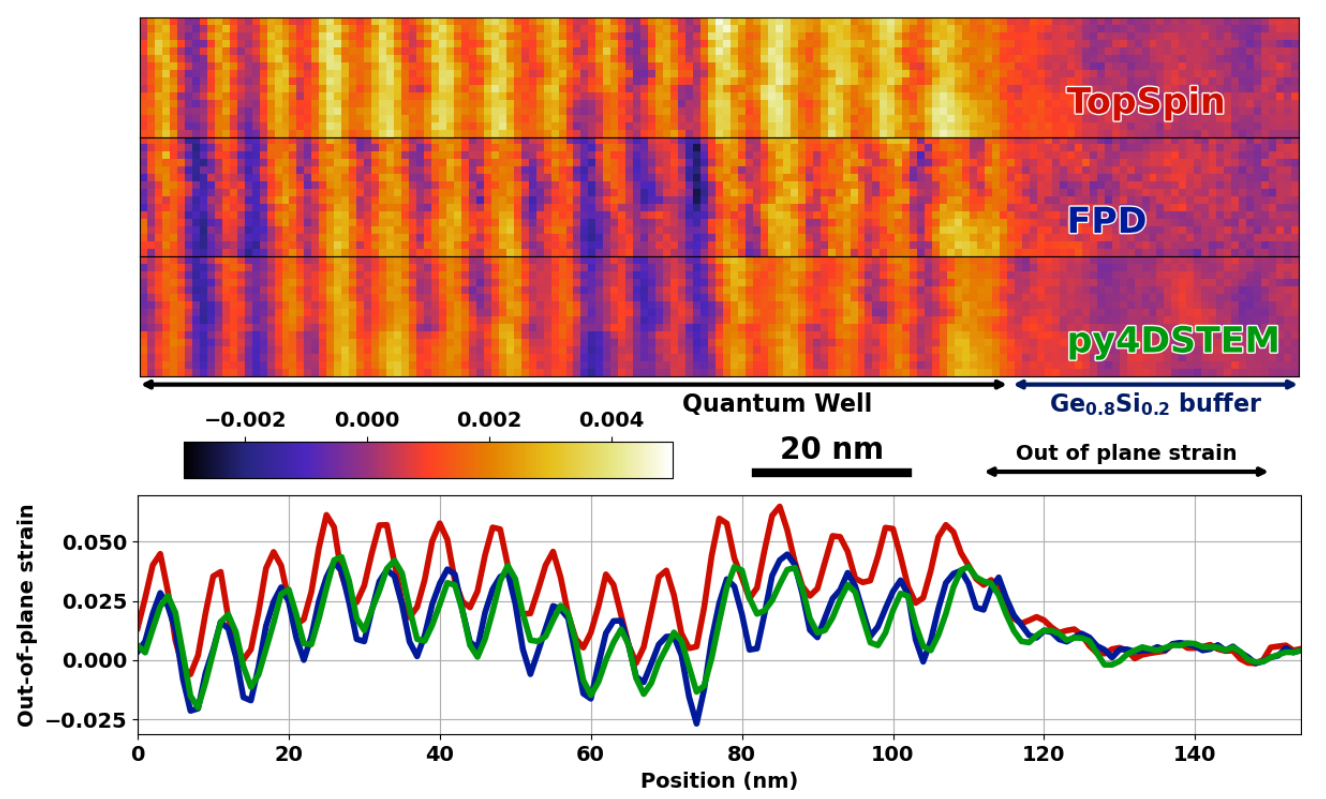

Figure 2. Out-of-plane strain maps calculated from the same dataset using three different software codes from an area of a Si:Ge/Ge quantum well structure on an 80:20 Ge:Si buffer, together with the averaged strain values plotted as a comparison graph below, showing some clear discrepancies between the different calculations.

References

1. A. Bashir, et al., J. Appl. Phys. 126, 235701 (2019).

2. A. D. Darbal, et al., Microsc. Microanal. 19, 702-703 (2013).

3. G. W. Paterson, et al., Microsc. Microanal. 26, 944-963 (2020).

4. B. H. Savitzky, et al., arXiv e-prints, arXiv:2003.09523 (2020).

5. R. Yuan, et al., Ultramicroscopy 207, 112837 (2019).

6. E. Padgett, et al., Ultramicroscopy 214, 112994 (2020).

7. A. Bashir, et al., J. Appl. Phys. 123, 035703 (2018). 